\title{
Evaluating the effectiveness of using enoxaparin to prevent venous thromboembolism in hip replacement patients: $A$ retrospective cohort study
}

\author{
Vo Thanh Toan ${ }^{1, *(}$, To Dong Kha ${ }^{\circledR}$, Bui Van Anh ${ }^{1 \oplus}$
}

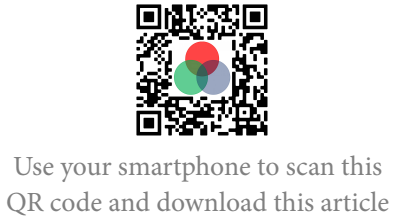

${ }^{l}$ Department of Orthopedic Surgery, Thong Nhat Hospital, Ho Chi Minh City, Vietnam

${ }^{2}$ School of Medicine, Vietnam National University Ho Chi Minh City, Ho Chi Minh City, Vietnam

Correspondence

Vo Thanh Toan, Department of Orthopedic Surgery, Thong Nhat Hospital, Ho Chi Minh City, Vietnam

Email: vothanhtoan1990@yahoo.com

History

- Received: 2021-12-05

- Accepted: 2021-02-05

- Published: 2021-02-28

DOI : 10.15419/bmrat.v8i2.661

\section{Check for updates}

Copyright

( $)$ Biomedpress. This is an openaccess article distributed under the terms of the Creative Commons Attribution 4.0 International license.

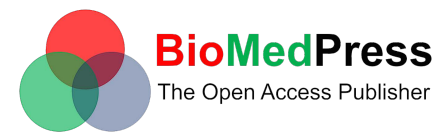

\begin{abstract}
Introduction: Our research aims to evaluate the effectiveness of thromboprophylaxis using enoxaparin in patients undergoing hip replacement. Methods: A retrospective cohort study was conducted based on medical records of patients aged 40 years and older undergoing hip replacement. Exclusion criteria included patients who had used anticoagulants to prevent other diseases, patients with a history of chronic renal failure, liver failure, cancer or allergy to anticoagulants, and patients with indicated mechanical prophylaxis. In our study, 65 patients were randomized into 2 groups - the control group and the venous thromboembolism (VTE) prophylaxis group (receiving subcutaneous enoxaparin $40 \mathrm{mg}$ daily for 7 - 14 days). Preventive effectiveness was evaluated based on the comparison of VTE incidence after surgery between the groups. Results: In our study, most of the patients were over 60 years old (79.2\%). No case of pulmonary embolism was recorded. There were 11 patients in the control group (17.2\%) who developed deep venous thrombosis (DVT) versus 2 patients in the prophylaxis group (3.1\%). After adjusting for postoperative hospital stay, use of enoxaparin reduced the risk of DVT by $89.7 \%$ (OR 0.103, 95\% confidence interval $0.019-0.569, \mathrm{p}$ $=0.009$ ), especially in patients over 60 years old (OR 0.147, 95\% confidence interval $0.026-0.822$, $p=0.029)$. Conclusion: This study demonstrates that using enoxaparin significantly reduces the incidence of DVT in patients undergoing hip replacement, especially in patients over 60 years old. Key words: Venous thromboembolism, Hip replacement, Enoxaparin, VTE prophylaxis, Deep venous thrombosis
\end{abstract}

\section{INTRODUCTION}

Venous thromboembolism (VTE) is currently a threat to surgical patients and a burden to health care systems around the world. In the United States, there are 900,000 new cases and nearly 300,000 deaths related to VTE each year ${ }^{1}$. In particular, hip replacement surgery is a high-risk factor for VTE. After total hip replacement surgery, without any preventive measures, the incidence of deep vein thrombosis (DVT) and pulmonary embolism (PE) are $42-57 \%$ and $0.9-28 \%$, respectively, and after partial hip replacement surgery, the rates are $46-60 \%$ and $3-$ $11 \%$, respectively ${ }^{2}$. So far, there have been many international studies showing the effectiveness of VTE prophylaxis in patients undergoing hip replacement surgery. Many VTE prophylaxis guidelines have been issued, such as those from the American Society of Thoracic Doctors (ACCP) which have been widely used in North America and around the world over the past 25 years, for patients undergoing joint replacement surgery ${ }^{2}$. In Vietnam, VTE prophylaxis guidelines after hip and knee replacement surgery were rec- ommended by the Ho Chi Minh City Orthopedic Association in $2013^{3}$. However, at Thong Nhat Hospital, the prevention of VTE with low molecular weight heparin (enoxaparin) for hip replacement surgery patients has recently been implemented. Thus, research is needed to assess the effectiveness of this preventive method.

\section{MATERIALS - METHODS}

The study was conducted based on medical records of patients aged 40 and older who had hip replacement surgery at Thong Nhat Hospital from January 2014 to January 2017. The exclusion criteria included those patients with a medical record stating that they had previously used anticoagulation to prevent other medical conditions, those with a history of chronic renal failure, liver failure, cancer, or allergy to anticoagulants, and those prescribed with mechanical prophylaxis method after surgery. Based on a study by Yoo et al. on the prophylaxis effect of low molecular weight heparin (LMWH) on VTE in Asian patients ${ }^{4}$, we calculated the required sample size to be 
130 patients, which includes 65 patients in the nonprophylactic group (no anticoagulant indicated) and 65 patients in the prophylactic group (indicated for enoxaparin (LOVENOX ) subcutaneously at a dose of $40 \mathrm{mg} /$ day for 7 - 14 days).

This was a retrospective cohort study. The medical records were randomly selected from 2 groups of patients using MS Excel 2010 software. For each medical record, information was obtained on patient characteristics (age, gender, weight, height, length of hospital stay, reasons for admission, medical history, and allergy history), surgical characteristics (cause, surgical choice, length of surgery, and presence or absence of indication of VTE prophylaxis with enoxaparin), and postoperative follow-up (clinical signs of DVT, $\mathrm{PE}$, and subclinical tests). The diagnostic criteria for VTE followed the recommendations of the American Thoracic Association (ACCP) in 2012 and those from the American Academy of Orthopedic Surgery (AAOS) in $2011^{5}$. Patients were considered to have VTE after surgery if they showed clinical manifestations associated with the definitive diagnosis of DVT (based on the results of leg vein Doppler ultrasound) or the diagnosis of PE (based on chest CT scan).

Data were collected and processed statistically by SPSS 20.0 software with $95 \%$ confidence. Variables with normal distribution are presented as mean \pm standard deviation; variables with non-standard distribution are presented by median. Descriptive statistics were used to calculate the percent, mean, and median of variables. The Student's t-test was used to compare the average of variables according to the normal distribution. In the case of a variable with a nonstandard distribution, the Mann-Whitney U test was used. The chi-square test was used to compare percentages between the two groups if the expected average value in the cells was $\geq 5$. The Fisher test was used when at least 1 cell had the expected value $<5$. Regression logistic was used to correct for confounding variables (if any). P-value $<0.05$ was considered to represent a statistically significant difference.

\section{RESULTS}

In the 130 records surveyed, men accounted for the majority of the subjects $(55.4 \%)$. The median age of patients in the study was $78(40-99)$, of which patients over 60 years accounted for $79.2 \%$. Patients were weighed and calculated by body mass ratio (BMI), using BMI classification for Asians ${ }^{6}$, except for some patients with severe femoral neck fractures with advanced age or those too weak to be measured. The average BMI of the 109 patients weighed (60 patients in the non-prophylaxis group and 49 patients in the prophylaxis group) was $21.3 \pm 3.2 \mathrm{~kg} / \mathrm{m}^{2}(12.5$ - 27.8). The reported comorbidities included hypertension, osteoarthritis, diabetes, surgical intervention, chronic obstructive pulmonary disease (COPD), and other conditions (e.g. dyslipidemia, chronic gastritis, hypertrophy, benign prostate, gout, dementia, and Parkinson's). The predominant causes leading to hip replacement surgery included femoral neck fracture $(83.8 \%)$ and hip degeneration (16.2\%). Moreover, $79.2 \%$ of patients had a partial hip replacement and $20.8 \%$ had a total hip replacement. The comparison of the population characteristics of the nonprophylaxis and prophylaxis groups are presented in Table 1.

In our study, no postoperative PE was recorded. A total of 16 patients developed symptoms of DVT, of which 14 were diagnosed with DVT based on a positive result for thrombosis by venous Doppler ultrasound. The symptoms noted in these 14 patients included an increased local sensation of heat (13/14 cases), increased calf volume (14/14 cases), pain when touching the calf (10/14 cases), and ankle swelling (7/14 cases). In the non-prophylaxis group, there were 12 patients (18.5\%) who developed thrombosis after surgery. In particular, a 57-year-old patient with a complicated hospital infection had a prolonged hospital stay after surgery (103 days), limited mobility, and then was diagnosed with DVT on the 59th day. Excluding this case, the number of patients with DVT in the non-prophylaxis group was 11 out of 64 patients (17.2\%). The number of DVT patients in the prophylaxis group was recorded as 2 cases (3.1\%). Table 2 presents the comparison of DVT ratio between the two groups, with or without prophylaxis with enoxaparin, after adjusting for the confounding variable of postoperative hospital stay.

Moreover, during the treatment period, we recorded 2 cases of forced discontinued anticoagulation early on the 5th day of surgery. One was a 67-year-old man with a history of hypertension and hip osteoarthritis who quit treatment due to significant hematoma at the surgical incision. The other was a 79-year-old male with a history of myocardial ischemia, chronic colitis, and hemorrhoids who was discontinued due to bloody bowel movements.

\section{DISCUSSION}

The gender distribution in our study sample was quite similar as males accounted for $55.6 \%$ of the study population and females accounted for $44.6 \%$. Since most patients in our study were over 60 years of age (79.2\%), the study population was considered to be at high risk 
Table 1: Population characteristics of the $\mathbf{2}$ patient groups

\begin{tabular}{|c|c|c|c|}
\hline Characteristics & Non-preventive & Preventive & P value \\
\hline & $(n=65)$ & $(n=65)$ & \\
\hline \multicolumn{4}{|l|}{ Gender, $\mathbf{n}(\%)$} \\
\hline - Female & $27(41.5)$ & $31(47.7)$ & 0.48 \\
\hline - Male & $38(58.5)$ & $34(52.3)$ & \\
\hline \multicolumn{4}{|l|}{ Age group, $\mathbf{n}(\%)$} \\
\hline$-40-60$ years old & $14(21.5)$ & $13(20.0)$ & 0.829 \\
\hline$->60$ years old & $51(78.5)$ & $52(80.0)$ & \\
\hline \multicolumn{4}{|l|}{ BMI group ${ }^{\star}$, n (\%) } \\
\hline$-<18.5$ & $11(18.3)$ & $11(22.5)$ & 0.932 \\
\hline$-18.5-22.9$ & $30(50.0)$ & $24(49.0)$ & \\
\hline$-23.0-24.9$ & $12(20.0)$ & $8(16.3)$ & \\
\hline$-24.0-29.9$ & $7(11.7)$ & $6(12.2)$ & \\
\hline \multicolumn{4}{|l|}{ Comorbidities, $\mathbf{n}(\%)$} \\
\hline - Hypertension & $37(56.9)$ & $40(61.5)$ & 0.592 \\
\hline - Osteoarthritis & $11(16.9)$ & $11(16.9)$ & 1 \\
\hline - Diabetes & $7(10.8)$ & $13(20.0)$ & 0.145 \\
\hline - Surgical intervention & $5(7.7)$ & $6(9.2)$ & 0.753 \\
\hline - COPD & $1(1.5)$ & $2(3.1)$ & 0.559 \\
\hline - Other & $18(27.7)$ & $14(21.5)$ & 0.415 \\
\hline \multicolumn{4}{|l|}{ Surgical causes, n (\%) } \\
\hline - Femoral neck fracture & $55(84.6)$ & $54(83.1)$ & 0.812 \\
\hline - Hip degeneration & $10(15.4)$ & $11(16.9)$ & \\
\hline \multicolumn{4}{|l|}{ Types of surgery, n (\%) } \\
\hline - Partial hip replacement & $51(78.5)$ & $52(80.0)$ & 0.829 \\
\hline - Total hip replacement & $14(21.5)$ & $13(20.0)$ & \\
\hline \multicolumn{4}{|l|}{$\begin{array}{l}\text { Length of hospital stay (days), median } \\
\text { (range) }\end{array}$} \\
\hline - Preoperative & $5(1-33)$ & $4(1-65)$ & 0.194 \\
\hline - Postoperative & $15(8-35)$ & $19(7-51)$ & 0.017 \\
\hline Operative time (minutes), median (range) & $100(60-190)$ & $95(55-290)$ & 0.012 \\
\hline
\end{tabular}

${ }^{*}$ BMI classification for Asians ${ }^{6}$. Non-prophylaxis group $(n=60)$ and prophylaxis group $(n=49)$. 
Table 2: Distribution of patients with DVT

\begin{tabular}{lcccc}
\hline Group & Non-preventive & Preventive & $\begin{array}{c}\text { OR (95\% confidence } \\
\text { intervals) }\end{array}$ & P value \\
\hline Total, $\mathrm{n} / \mathrm{N}(\%)$ & $11 / 64(17.2)^{*}$ & $2 / 65(3.1)$ & $0.103(0.019-0.569)$ & 0.009 \\
$40-60$ years old, $\mathrm{n} / \mathrm{N}(\%)$ & $2 / 13(21.4)^{*}$ & $0 / 13(0)$ & 0.000 & 0.998 \\
$>60$ years old, $\mathrm{n} / \mathrm{N}(\%)$ & $9 / 51(17.6)$ & $2 / 52(3.8)$ & $0.147(0.026-0.822)$ & 0.029 \\
\hline
\end{tabular}

n: number of patients diagnosed with DVT

$N$ : Total number of patients

${ }^{*}$ Excluded 1 case of hospitalization period 103 days after surgery.

for $\mathrm{DVT}^{7}$. Our study results are consistent with a report on patients with hip replacement in Vietnam by Tran Trung Dung et al. ${ }^{8}$. We did not record any cases that were obese to the extent that the anticoagulant dose needed to be adjusted ${ }^{9}$. The predominant reason for surgery in the study herein was due to fracture of the femoral neck (83.8\%), and the majority of patients had partial hip replacement surgery (79.2\%). These results are consistent with the report by Tran Trung Dung et al. which found that $82.1 \%$ of patients had hip replacement due to a fracture of the hip bone, and the percentage of those having partial hip replacement was $61.5 \%{ }^{8}$. The duration of hospitalization before surgery of the two groups of patients in this study was higher than the duration of hospitalization before surgery in the SMART study ${ }^{10}$ (median 4-5 days compared to 2 days). Therefore, patients in our study were at higher risk for DVT compared to other studies when considering the factor of immobility before surgery.

Table 1 shows that the characteristics of patients in the two study groups are similar $(\mathrm{p}>0.05)$, except that the postoperative hospitalization period of the prophylaxis group is statistically higher than that of the non-prophylaxis group (median of 19 days compared to 15 days, $\mathrm{p}=0.017$ ). Thus, patients in the prophylaxis group with enoxaparin had more than one risk factor for DVT compared to the non-prophylaxis group. However, after hip replacement surgery, all patients at Thong Nhat Hospital were instructed to mobilize early; therefore, this difference may not affect the risk of VTE in the 2 groups. The results after adjustment of the confounding variable (the postoperative hospital stay) also demonstrated that the use of prophylactic enoxaparin VTE reduced $89.7 \%$ of the risk of DVT compared with not using any preventive measures (OR 0.103, 95\% confidence interval 0.019 $0.569, \mathrm{p}=0.009$ ).

The efficacy of enoxaparin on prophylaxis of DVT following hip replacement surgery in our study was consistent with previous studies conducted in Asian patients. In Korea, a 1997 study by M.C. Yoo et al. aimed to compare the prevalence of DVT between 2 groups (non-preventive or preventive with LMWH) after hip replacement of patients over 40 years of age. Their study showed a statistically higher incidence of DVT in the non-prophylaxis group ( $16 \%$ compared with $2 \%$ in the prophylaxis group, $\mathrm{p}=0.015)^{4}$. A $2001 \mathrm{co}-$ hort study by X.L. Li et al. in China on patients with undergoing hip and knee orthopedic surgery over 40 years old also showed that the rate of DVT in the nonprophylaxis group was higher than that of the prophylaxis group with LMWH (34.8\% compared with 4.3\%, $\mathrm{p}<0.05)^{11}$. Besides, other studies such as those by Turpie et al. (1986) ${ }^{12}$, Planes et al. (1996) ${ }^{13}$, Samama et al. (1997) ${ }^{14}$, have further demonstrated the efficacy of DVT prophylaxis using LMWH in hip replacement patients. Nearly $80 \%$ of patients in this study were over 60 years of age, a high-risk VTE age group. In the age group above 60 , the use of enoxaparin for prophylaxis of VTE after hip replacement surgery effectively reduces the risk of DVT by $85.3 \%$ compared to absence of prophylaxis (OR $0.147,95 \%$ confidence intervals $0.026-0.822, \mathrm{p}=0.029)$. These results suggest that enoxaparin administration for hip replacement patients over age 60 is essential to reduce the risk of post-surgery VTE.

\section{CONCLUSIONS}

Our study primarily showed that the use of enoxaparin to prevent venous thromboembolism in patients with hip replacement at Thong Nhat Hospital (Vietnam) was remarkably effective, especially in patients over 60 years old. However, future prospective randomized controlled trials with a larger number of patients are essential to confirm these preliminary findings.

\section{ABBREVIATIONS}

BMI: body-mass index

COPD: chronic obstructive pulmonary disease

DVT: deep vein thrombosis

LMWH: low molecular weight heparin

PE: pulmonary embolism

VTE: venous thromboembolism 


\section{ACKNOWLEDGMENTS}

The authors would like to thank Thong Nhat Hospital for granting permission to access medical records.

\section{AUTHORS' CONTRIBUTIONS}

All authors contributed in the study concept and design. VTT, TDK performed the data collection and analysis, all authors wrote the initial manuscript. VTT supervised the study and finalized the manuscript. All authors read and confirmed the publication of the article.

\section{FUNDING}

None

\section{AVAILABILITY OF DATA AND MATERIALS}

Data and materials used and/or analyzed during the current study are available from the corresponding author on reasonable request.

\section{ETHICS APPROVAL AND CONSENT TO PARTICIPATE}

This study was conducted in accordance with the amended Declaration of Helsinki. This research was approved by the Institutional Review Board of the Thong Nhat Hospital. (Project Number: 94 IRB/ QDBVTN 02042018).

\section{CONSENT FOR PUBLICATION}

Not applicable.

\section{COMPETING INTERESTS}

The authors declare that they have no competing interests.

\section{REFERENCES}

1. Heit JA, Cohen AT, Anderson FA. Estimated annual number of incident and recurrent, non-fatal and fatal venous thromboembolism (VTE) events in the US. Blood. 2005;106(11):910. Available from: https://doi.org/10.1182/ blood.V106.11.910.910.

2. Geerts WH, Bergqvist D, Pineo GF, Heit JA, Samama CM, Lassen MR, et al. Prevention of venous thromboembolism: American College of Chest Physicians evidence-based clinical practice guidelines. 2008;133(6 Suppl):381S-453S. PMID: 18574271. Available from: https://doi.org/10.1378/chest.08-0656.
3. Ho Chi Minh City Orthopedic Association, Viet Nam. Guidelines for VTE prophylaxis after hip and knee replacement surgery. 2013;

4. Yoo MC, Kang CS, Kim YH, Kim SK. A prospective randomized study on the use of nadroparin calcium in the prophylaxis of thromboembolism in Korean patients undergoing elective total hip replacement. Int Orthop. 1998;21(6):399402. PMID: 9498151 . Available from: https://doi.org/10.1007/ s002640050194.

5. Barrack RL. Current guidelines for total joint VTE prophylaxis: dawn of a new day. J Bone Joint Surg Br. 2012;94(11 Suppl A):3-7. PMID: 23118370. Available from: https://doi.org/10. 1302/0301-620X.94B11.30824.

6. Anuurad E, Shiwaku K, Nogi A, Kitajima K, Enkhmaa B, Shimono $K$, et al. The new BMI criteria for asians by the regional office for the western pacific region of WHO are suitable for screening of overweight to prevent metabolic syndrome in elder Japanese workers. J Occup Health. 2003;45(6):335-343. PMID: 14676412. Available from: https://doi.org/10.1539/joh. 45.335.

7. White RH. The epidemiology of venous thromboembolism. Circulation. 2003;107(23 Suppl 1):1-4. PMID: 12814979. Available from: https://doi.org/10.1161/01.CIR.0000078468.11849. 66.

8. Dung TT. Comment on traumatic characteristics and hip replacement surgery techniques at Hanoi Medical University Hospital. Clin Med (Northfield II). 2013;11:62-64.

9. Nutescu EA, Spinier SA, Wittkowsky A, Dager WE. Anticoagulation: low-molecular-weight heparins in renal impairment and obesity: available evidence and clinical practice recommendations across medical and surgical settings. Ann Pharmacother. 2009;43(6):1064-1083. PMID: 19458109. Available from: https://doi.org/10.1345/aph.1L194.

10. Leizorovicz A, Turpie AGG, Cohen AT, Wong L, Yoo MC, Dans A. Epidemiology of venous thromboembolism in Asian patients undergoing major orthopedic surgery without thromboprophylaxis. The SMART Study. J Thromb Haemost. 2005;3(1):2834. PMID: 15634263 . Available from: https://doi.org/10.1111/j. 1538- 7836.2004.01094.x.

11. Li XL, Lu WJ, Yu NS. Prophylaxis for deep vein thrombosis with low molecular weight heparin following hip and knee surgery. Zhongguo Xiu Fu Chong Jian Wai Ke Za Zhi= Zhongguo Xiufu Chongjian Waike Zazhi Chinese J Reparative Reconstr Surg. 2001;15:39-41.

12. Turpie AGG, Levine MN, Hirsh J, Carter CJ, Jay RM, Powers PJ, et al. A randomized controlled trial of a low-molecularweight heparin (enoxaparin) to prevent deep-vein thrombosis in patients undergoing elective hip surgery. N Engl J Med. 1986;315(15):925-929. PMID: 3531851. Available from: https: //doi.org/10.1056/NEJM198610093151503.

13. Planes A, Vochelle N, Darmon J-Y, Fagola M, Bellaud M, Huet Y. Risk of deep-venous thrombosis after hospital discharge in patients having undergone total hip replacement: doubleblind randomised comparison of enoxaparin versus placebo. Lancet. 1996;348(9022):224-228. Available from: https://doi. org/10.1016/S0140-6736(96)01453-5.

14. Samama CM, Clergue F, Barre J, Montefiore A, III P, Samii K. Low molecular weight heparin associated with spinal anaesthesia and gradual compression stockings in total hip replacement surgery. Arar Study Group. Br J Anaesth. 1997;78(6):660-665. PMID: 9215015 . Available from: https://doi.org/10.1093/bja/78. 6.660. 